\title{
COMPLEX ORTHODONTIC-IMPLANT TREATMENT IN INCISOR HYPODONTIA CASES
}

\author{
Miroslava Dinkova, \\ Department of Orthodontics, Faculty of Dental Medicine, Medical University - \\ Sofia, Bulgaria.
}

\section{SUMMARY:}

Purpose: The purpose of the current paper is to show clinical cases with unilateral and bilateral incisal hypodontia, treated with complex orthodontic - implant treatment.

Materials and methods: 3 representative clinical cases are included with interdisciplinary complex treatment protocol attached: case 1 - asymmetric upper right lateral incisor hypodontia, case 2 - symmetric upper lateral incisors hypodontia and case 3 - symmetric lower central incisor hypodontia. Cases are followed over ten and a half and eight years retention period.

The interdisciplinary treatment protocol includes the following stages:

First stage: Pretreatment preparation to form a crew, planning the type of the manipulations, sequence and duration, finance plan, discussion of the treatment plan and alternative options with the patient, signed informed consent.

Second stage: Orthodontic treatment.

Third stage: Implant restorations.

Fourth stage: Aesthetic prosthetic restorations. Retention period.

Results: In all three cases, aesthetics were improved. We obtained the correct ratio of the upper lip to the dental arch. Paralleling the roots of the frontal teeth and their levelling up provided correct, balanced occlusal and proximal contacts, which improved the function and provided stability of the treatment results.

Conclusion: The complex orthodonticimplantology approach for the treatment of single tooth hypodontia the tooth arch is successful and ensures optimal and lasting restoration of function and aesthetics of the dental-jaw system.

Keywords: hypodontia, orthodontics, dental implant, braces

\section{INTRODUCTION:}

Hypodontia is frequently seen tooth anomaly in the permanent dentition, which is most often presented in lack of upper second incisors, lower second premolars and lower central incisors.

The difficulties in treating hypodontia of the permanent incisors are related to the reduction of the alveolar bone in the affected area, spaces between the teeth, asymmetry, lack of space for the missing tooth, worsened aesthetics. [1] Choosing a treatment plan depends on the presented malocclusion, the size of the teeth, occlusion, age and aesthetics. In consideration with the contemporary tendencies for function and aesthetics, the clinical protocol which includes orthodontic treatment for creating space and restoring the missing teeth, prevails. [2, 3]

The high frequency and difficulties when treating hypodontia defined the need for setting up an interdisciplinary approach when planning and conducting the treatment.

\section{MATERIALS AND METHODS:}

Three representative clinical cases are included with interdisciplinary complex treatment protocol attached: case 1 - asymmetric upper right lateral incisor hypodontia, case 2 - symmetric upper lateral incisors hypodontia and case 3 - symmetric lower central incisor hypodontia. Cases are followed in over ten and a half and eight years retention period.

The interdisciplinary treatment protocol includes the following stages:

First stage: Pretreatment preparation to form a crew, planning the type of the manipulations, sequence and duration, finance plan, discussion of the treatment plan and alternative options with the patient, signing an informed consent.

Second stage: Orthodontic treatment.

Third stage: Implant restorations.

Fourth stage: Aesthetic prosthetic restorations. Retention period. 


\section{Clinical case 1:}

Patient L.S., 21 years old with asymmetric upper right lateral incisor hypodontia, Dental class I, dislocation of 11 left ( $3 \mathrm{~mm})$, medialized 13 , rotation of 13,23 , retroclined incisors, diastema $3 \mathrm{~mm}$, tremas $(11 / 13-1,5$ $\mathrm{mm}, 13 / 14-2,0 \mathrm{~mm}, 23 / 24-1 \mathrm{~mm}$ ), overjet $0 \mathrm{~mm}, 1 \mathrm{~mm}$ overlap in the front segment, midline mismatch, normodivergent type, low attached upper lip frenulum. The orthopantomography confirmed the lack of upper right lateral incisor. (fig.1) Orthodontic treatment was conducted in upper dental arch using Inspire Ice, Roth .018" aesthetic brackets. Duration of treatment -17 months.

Fig. 1. Case 1 - Hypodontia 12: a, b. Extraoral photo-documentation before the orthodontic treatment and after the complex treatment; c. Intraoral photo-documentation before orthodontic treatment; d. Set-up and planning of orthodontic treatment; e. After complex treatment - eight years of retention.
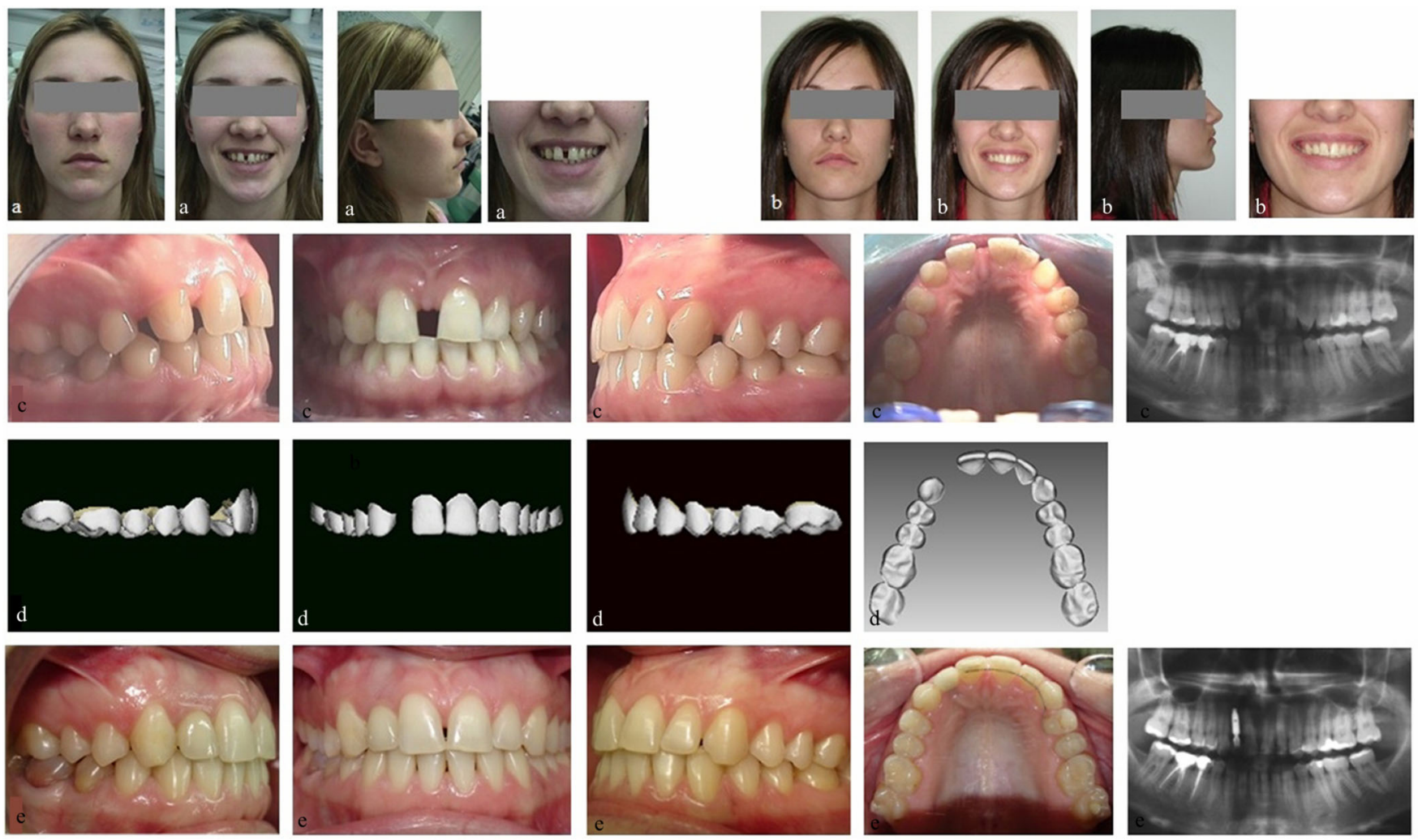

Orthodontic treatment included: medialization of 11 until closing the diastema and improving the symmetry; closing the trema between 13 and 14 with distal movement of 13; derotation of 13, 23, aligning of 23; paralleling 13 and 11 ; creating space for 12 with the intention of subsequent implantology; retention using fixed retainer after definitive restoration of the implant-supported superstructure.

Surgical implant treatment included taking the face bow values with the orthodontic appliance on and placing the casts in articulator with the individual values. Set up a Wax-up on the stone casts, define the direction of the implant and abutment using computer tomography. Placing an implant Ankylos with $11 \mathrm{~mm}$ length and diameter $-3,5 \mathrm{~mm}$ by preparation of a mucoperiosteal flap. The brackets were left passively. Temporary plastic tooth - spaceguard was laboratory-made for aesthetics. The tooth was fixed to the orthodontic archwire 10 days after

the surgical intervention. Sulcus-former was placed for gingival shaping 5 months later and straight abutment (4 $\mathrm{mm}$ ) after a 20 days interval.

For aesthetic prosthetic restoration, a definitive crown type EMPRESS 2 - L was made, fixed permanently to the abutment a month later.

\section{Clinical case 2:}

Patient I.Z., 18 years old, with persisted 71, symmetrical lower central incisor hypodontia, dental class I, rotated and inclined teeth $12,22,32,42$, tremas 32/42 $12,1 \mathrm{~mm}$, diatema $2,5 \mathrm{~mm}$, overjet $0 \mathrm{~mm}, 3,5 \mathrm{~mm}$ overlap in the front segment, skeletal class I, hypodivergent type. Orthopantomography confirmed the lack of germs of the lower central incisors. Lack of the needed parameters was measured in the alveolar crest in the region of 31,41 for implant restorations. (fig.2) 
Fig. 2. Case 2 - Hypodontia 31 and 41: a, b. Extraoral photo-documentation before orthodontic and after complex treatment; c. Intraoral photo-documentation before orthodontic treatment; d. Medialisation of 32, 42, creation of orthodontic induced bone tissue in the field of implantation; e. After complex treatment; f. Five years of retention.
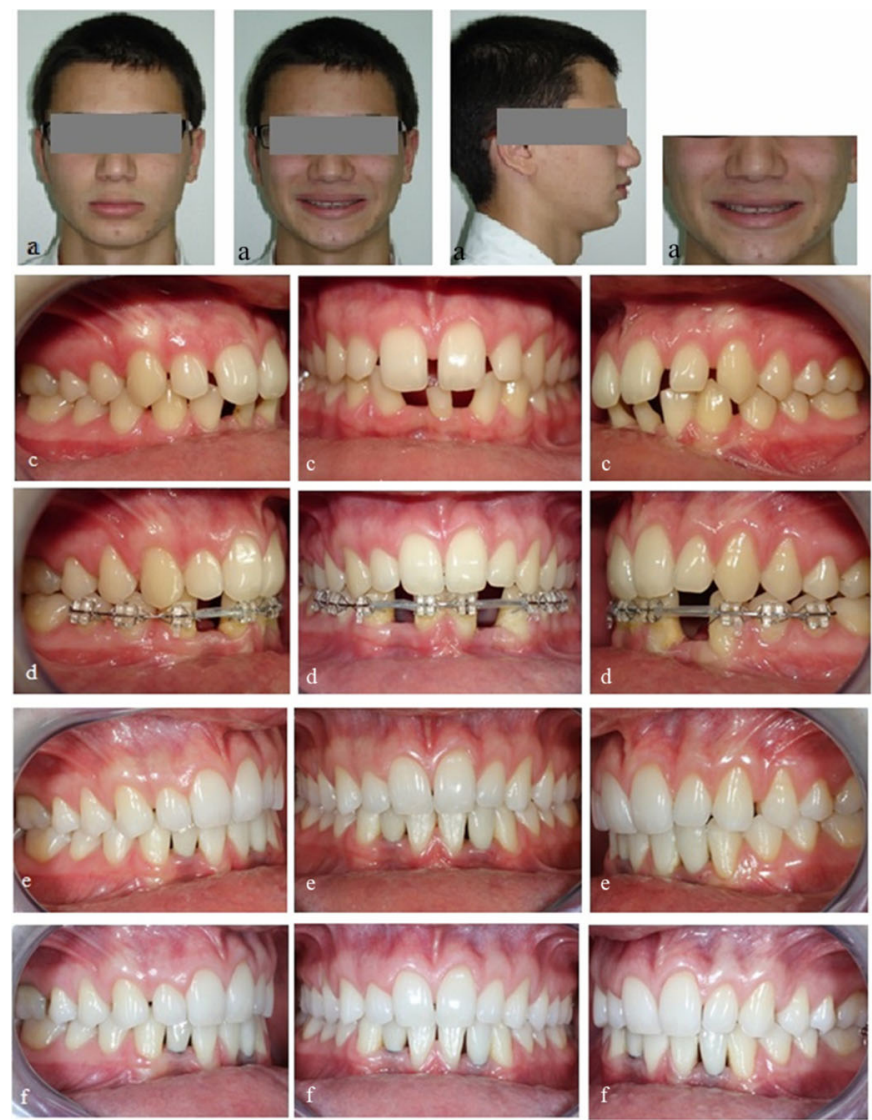

Complex interdisciplinary treatment was conducted using the described protocol.

Full orthodontic treatment was conducted with lingual STb brackets in the upper dental arch and aesthetic vestibular brackets - Inspire Ice Roth .018" in the lower dental arch. Duration of treatment -26 months. After the extraction of 71, teeth in upper and lower dental arches were levelled up, 32 and 42 are medialized until proximal contact has been established and an incisal point, which coincided with the midline of the lower jaw, distal root tipping and derotation of 33, 43, space has been created for single implants in the region of 32 and 42. Retention: fixed retainers in the upper and lower jaw. The retention in the lower jaw has been straightened with a vacuum retainer.

The implants have been placed in region 32, 42 Bicon 260-335-011 with 11,0 mm length and diameter -
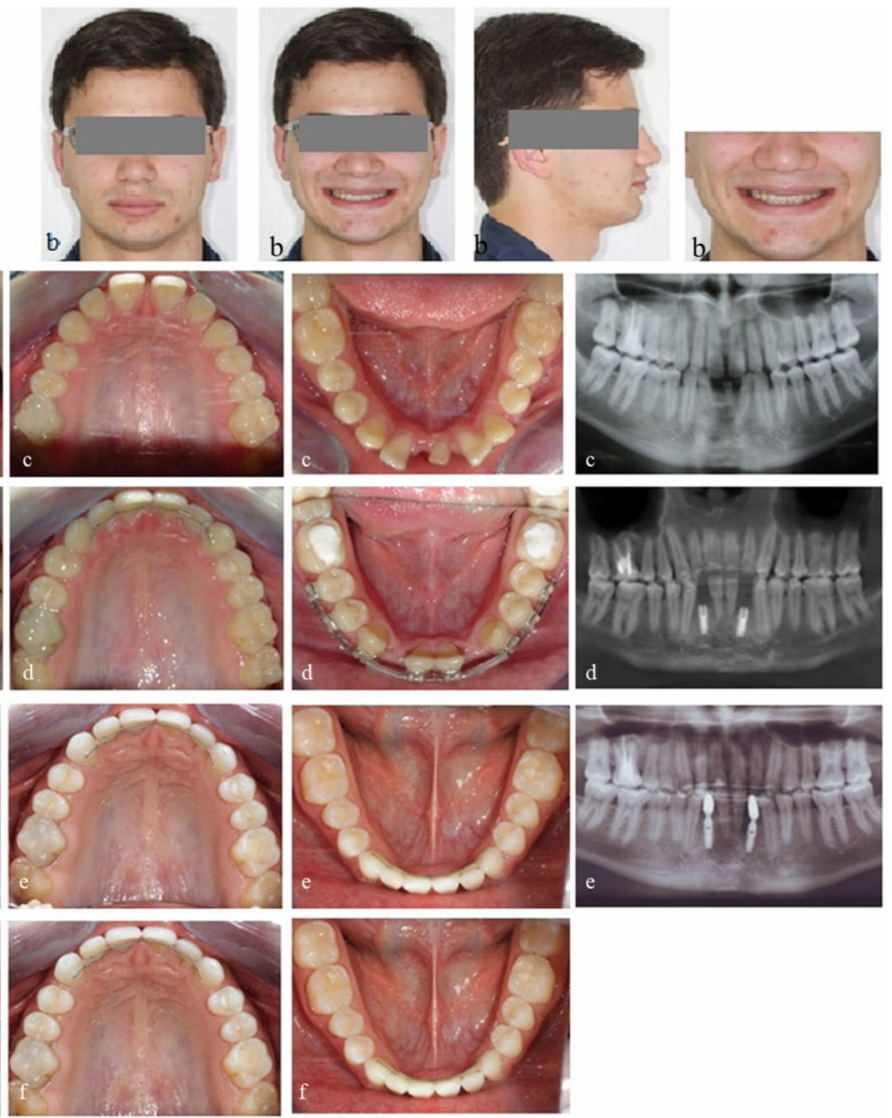

3,5 mm Integra - CP, Implant 2,0 $\mathrm{mm}$, Well by preparation of a mucoperiosteal flap. Spaceguards were fixed (32 and 42) to the previously made orthodontic retainer of lower frontal teeth. Aesthetic crowns were adjusted on the implants after osteointegration.

\section{Clinical case 3:}

Patient M.S., 13 years old with symmetrical bilateral hypodontia of 12,22 , dental class I, persisted 53, a medial eruption of 13,23 , tremas $(11 / 13-1 \mathrm{~mm}, 21 / 23$ $1 \mathrm{~mm}, 23 / 24-2,5 \mathrm{~mm})$.

After conducting the first stage, metal brackets Victory, Roth .018" were placed for levelling up the upper dental arch, distalization of 13,23, paralleling the axes of the roots of 11,13 and 21,23 , moving the midline of the upper dental arch to the right and restoring the symmetry. (fig. 3) 
Fig. 3. Case 3 - Hypodontia of 12, 22: a, b. Extraoral photo-documentation before and after complex treatment; c. End of orthodontic treatment completed with a fixed retainer and space guards of 12 and 22; d. Relapse after the remodelling of the fixed retainer because of the patient's decision after the first treatment. Clinical rotations of teeth are observed. The orthopantomography shows the converging apical longitudinal axes of 13, 11 and $21,23$. Re-orthodontic treatment for mesial root tipping of 11, 21 and distal root tipping of 13, 23; e. Orthodontic dental arch preparation for implantology; f. Aesthetic restoration of upper dental arches with single implants at 12 and 22.
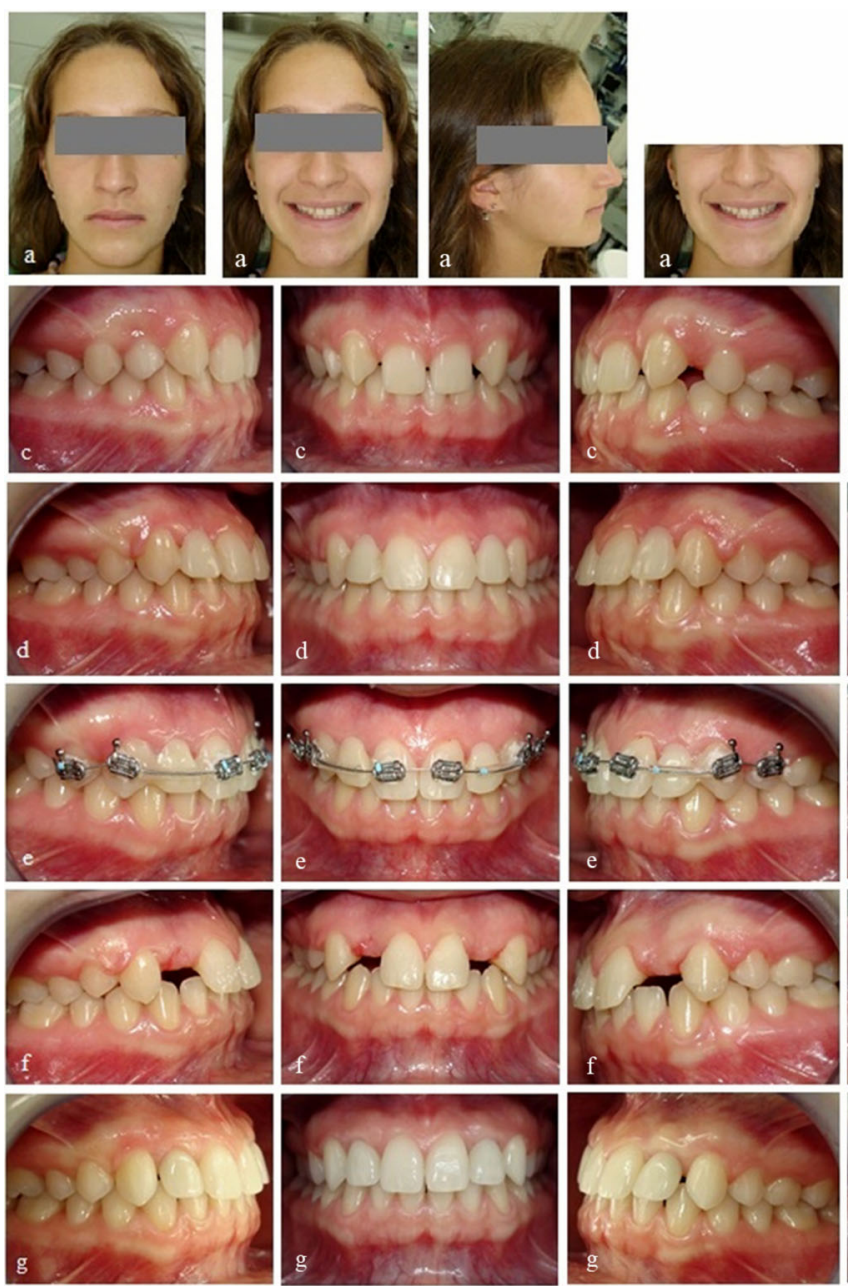

RESULTS:

In case 1 symmetry was achieved in an upper dental arch, 7,25 mm space was provided for implant at 12, spaces between teeth were closed, midline coincided, smile line was improved.

In case 2 were achieved 14,1 $\mathrm{mm}$ in the perimeter of the dental arch for implanting of single implants in the area of 32,42 . The roots of $33,43,32,42$ were parallelized. Midlines and incisal points were coincided in the upper and lower dental arches. 1/3 overlap of the clinical crown in the front was achieved. Height and buccolingual width of the alveolar crest was provided, which allowed the implanting of single implants in the region of 32,42 .

In case 3 upper permanent canines were moved in
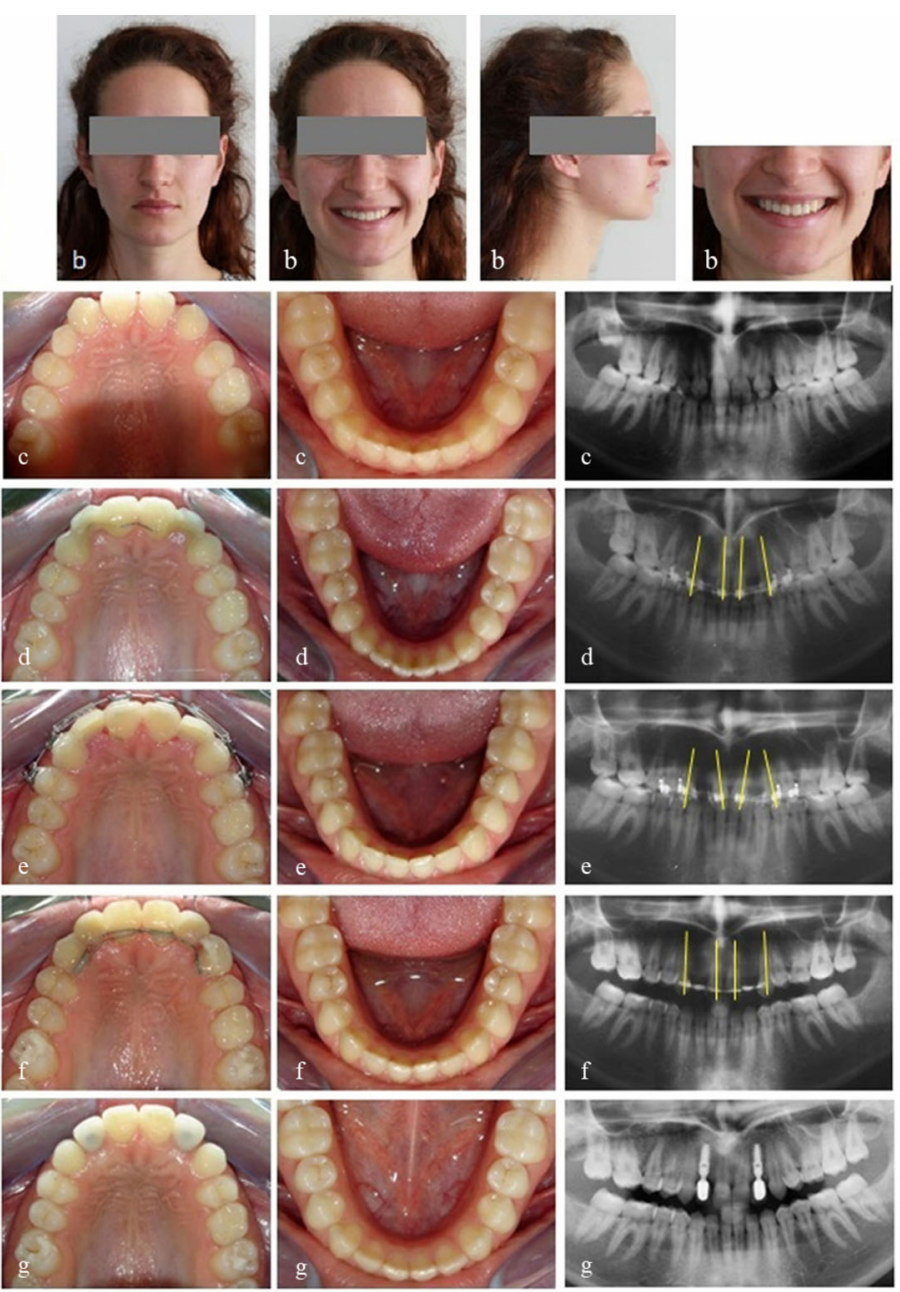

the correct position, with parallel ratios of the longitudinal axes of 11, 13 and 21,23. 7,00 $\mathrm{mm}$ and 7,30 $\mathrm{mm}$ space was provided for in the perimeter of the dental arch for the implantation of 12 and 22, symmetry was achieved in the upper frontal region.

In all three cases, aesthetics was improved. We obtained the correct ratio of the upper lip to the dental arch. Paralleling the roots of the frontal teeth and their levelling up provided correct, balanced occlusal and proximal contacts, which improved the function and provided stability of treatment results.

\section{DISCUSSION:}

Congenital lack of permanent incisors is an aesthetical and functional problem. Conditions for move- 
ment and inclination of adjacent teeth are created, alveolar bone is undeveloped with not enough buccolingual width, in the area of the hypodontia, lack of attached gingiva, lip frenulum is occlusally attached.

In these cases, restoration with the aesthetic bridge is difficult. Preparing intact teeth to be bridge holders is a compromise treatment option. [4]

With a treatment plan involving implantology, there are two approaches: bone grafting (1) or tooth displacement, the toothless area and placement of implants where teeth would have erupted normally. The orthodontic displacement of teeth through a toothless area due to extraction or congenital absence leads to restoration of the alveolar structures and parameters of the alveolar ridge. $[5,6,7]$

Cortical alveolar bone in front of the tooth is preserved with slow, light force movements through healthy gingival tissues. The periosteum of the buccal and lingual wall of the alveolus stimulates the formation of bone tissue through an apposition, creating healthy complete tissues for implantology. Fast movement and/or unsatisfactory oral hygiene can lead to dehiscence and compromise of treatment. [5, 8]

According to Kokich et al. after extraction, the buccolingual width in the anterior jaw section decreases by $23 \%$ in the first 6 months, and another $11 \%$ after 5 years, i. e. a total of about $34 \%$ over 5 years. This conclusion is decisive in the decision to extract the persist- ent teeth in case of hypodontia.

By reinforcing the alveolar ridge upon orthodontic displacement of the teeth through a toothless region, the resorptive changes are negligible, as observed in clinical cases 1 and 2. [9, 10, 11]

The particularities of the retention period are mainly related to the time for implantology and the type of retainers used in consideration of the subsequent orthodontic complete sanitation. The fixed retainer, with a maximum number of teeth included for sufficient rigidity, provides reliable retention after active orthodontic treatment. Under the action of the bite forces, a relapse is possible, expressed in the apical tipping of the teeth to the implant gap, which could reduce the width provided for it, shown in case 3, with an insufficiently stable retainer. The removable retainer does not provide the required retention of the achieved results. Its application is shown to enhance the stability of the fixed retainer. The removable vacuum retainer provides free access to the surgical field for implantology without risk of tooth displacement. [5, 12, 13]

\section{CONCLUSION:}

The complex orthodontic-implantology approach for the treatment of single tooth hypodontia in the tooth arch is successful and ensures optimal and lasting restoration of function and aesthetics of the dental-jaw system.

\section{REFERENCES:}

1. Robertson,S, Mohlin B. The congenitally missing upper lateral incisor: A retrospective study of orthodontic space closure versus restorative treatment. Eur J Orthod. 2000 Dec;22(6):697-710. [PubMed] [Crossref]

2. Bozkaya E, Canigur BN, \& Ulasan B. New perspective for evaluation of tooth widths in patients with missing or peg-shaped maxillary lateral incisors: Quadrant analysis. Am J Orthod Dentofacial Orthop. 2018 Dec;154(6):820-828. [ [PubMed] [Crossref]

3. Yordanova G. Tooth agenesis the problem and its solving in our practice, prevalence and relation with other deformities. J of IMAB. 2015

\section{Sep;21(3):859-863 [Crossref]}

4. Hunstadbraten K. Hypodontia in the permanent dentition. ASDC J Dent Child. 1973 Mar-Apr;40(2):115-7. [PubMed]

5. Kokich VO Jr. Congenitally missing teeth: orthodontic management in the adolescent patient. Am J Orthod Dentofacial Orthop. 2002 Jun;121(6):594-5. [PubMed] [Crossref]

6. Abu-Hussein M, Abdulgani A, Watted N, Zahalka M. Congenitally Missing Lateral Incisor with Orthodontics, Bone Grafting and Single-Tooth Implant: A Case Report. J Dent Med Sci (IOSR-JDMS). 2015 Apr;14(4)Ver.VI:124-130.

7. Paduano S, Cioffi I, Rondo R,
Cupo A, Bubbi R, Valletta R. Orthodontic Management of Congenitally Missing Maxillary Lateral Incisors: A Case Report. Case Rep Dent. 2014;2014:731074. [PubMed] [Crossref]

8. Muhamad AH, Nezar W, Azzaldeen A. Managing congenitally missing lateral incisors with single tooth implants. Dent Oral Craniofac Res. 2016 Aug;2(4):318-324. [Crossref]

9. Fekonja A. Hypodontia in orthodontically treated children. Eur J Orthod. 2005 Oct;27(5):457-60. [PubMed] [Crossref]

10. Ingervall B, Seeman L, Thilander B. Frequency of malocclusion and need of orthodontic 
treatment in 10-year old children in Gothenburg. Sven Tandlak Tidskr. 1972 Jan;65(1):7-21. [PubMed]

11. Garg AK. Treatment of congenitally missing maxillary lateral incisors: Orthodontics, bone grafts and osseointegrated implants. Dent Implantol Update. 2002 Feb;13(2):9-

\section{4. [PubMed]}

13. Antonarakis GS, Prevezanos P,

12. Richardson G, Rossell KA. Gavric J, Christou P. Agenesis of Congenitally missing maxillary lateral incisors and orthodontic treatment considerations for the single-tooth implant. J Can Dent Assoc. $2001 \quad$ Jan;67(1):25-8. maxillary lateral incisor and tooth replacement: cost-effectiveness of different treatment alternatives. Int $J$ Prosthodont. 2014 May-Jun;27(3): Jan;67(1):25-8. 257-63. [PubMed] [Crossref]

[PubMed]

Please cite this article as: Dinkova M. Complex orthodontic-implant treatment in incisor hypodontia cases. $J$ of IMAB. 2020 Jan-Mar;26(1):2892-2897. DOI: https://doi.org/10.5272/jimab.2020261.2892

Received: 24/04/2019; Published online: 06/02/2020

\section{Address for correspondence:}

Miroslava Dinkova

Associate professor in the Department of Orthodontics, Faculty of Dental Medicine, Medical University - Sofia,

St. Jerusalem 6, fl. 4, office 10, Sofia, Bulgaria

E-mail: miroslavadinkova@abv.bg 